\title{
Mechanical Properties of Hydroxyapatite Composites Reinforced with Hydroxyapatite Whiskers
}

\author{
D. Şimşek, R. Çiftçioğlu, M. Güden, M. Çiftçioğlu, Ş. Harsa \\ Materials Science and Engineering Program, \\ İzmir Institute of Technology, Gülbahçe Urla, İzmir
}

Keywords: Composite, Hydroxyapatite, Whisker, Sintering behaviour, Mechanical properties.

\begin{abstract}
Sintering and mechanical behavior of pure and hydroxyapatite (HA) whisker reinforced HA composites were investigated in this work. Pure and composite samples were prepared by using a commercial powder and whiskers prepared by molten salt synthesis. The dry-pressed samples were sintered in the 800 and $1300^{\circ} \mathrm{C}$ range. The effect of whisker-addition on the mechanical properties of HA was investigated through compression and hardness testing. Compressive strength and fracture strain were observed to increase by the addition of whiskers.
\end{abstract}

\section{Introduction}

Although HA is one of the most biocompatible materials, the mechanical reliability and fracture toughness of pure HA ceramics is low. The purpose of preparing HA composites with biocompatible HA whiskers is to enhance the mechanical properties of pure HA ceramics [1]. The mismatch of mechanical properties may cause the stress concentration in the bone - implant interface that may consequently end up with the death of the surrounding tissue especially in loadbearing operations besides biocompability. The bone must be under a certain amount of load in order to remain healthy. Weakening of the bone and degradation of the bone-implant interface along with possible biological changes may occur if the bone is unloaded or overloaded in compression [2]. The effects of molten salt synthesised HA whiskers at 10-30 vol \% on the mechanical properties of Ha ceramics was investigated in this work

\section{Materials and Method}

Commercial HA powder (Aldrich) was used to prepare pure HA and HA whisker reinforced composites. $\mathrm{HA}$ whiskers were prepared by molten salt synthesis at $1100^{\circ} \mathrm{C}$ with a $\mathrm{HA} / \mathrm{K}_{2} \mathrm{SO}_{4}$ ratio of 3 (the melting point of $\mathrm{K}_{2} \mathrm{SO}_{4}: 1060{ }^{\circ} \mathrm{C}$ ) [3]. Composites with 10-30 vol \% whisker content were prepared by the addition whiskers in to high solid loaded HA suspension with ultrasonic treatment. The mixture was then oven dried at $90{ }^{\circ} \mathrm{C}$. Composite powders and pure HA powder were compacted in a cylindrical die under a pressure of $160 \mathrm{MPa}$. The relative density of the pellets was about 55\%. Pure and composite HA pellets were finally sintered in the 800 and $1300{ }^{\circ} \mathrm{C}$ temperature range. Compression testing of the sintered samples, $8 \mathrm{~mm}$ in diameter and $4 \mathrm{~mm}$ in height, were performed by a Testometric M500-100kN universal-testing machine with a crosshead speed of $0,5 \mathrm{~mm} / \mathrm{min}$. The Vickers hardness tests (Time HVS-1000) were conducted under $4.9 \mathrm{~N}$ load with $20 \mathrm{sec}$ loading time. Scanning electron microscopy (Philips XL30 SFEG) and XRD (Philips E'XPERT PRO) techniques were used to characterised powders and ceramic samples.

\section{Results and Discussion}

The XRD patterns of the commercial HA powder and the HA whiskers are shown in Figure 1(a) and (b). The similarity between the XRD patterns of the HA whisker and commercial HA powder confirms that $\mathrm{HA}$ is the dominant phase in whisker. The intense and narrow peaks in Figure 1(b) 
especially in the (300) plane shows the preferential growth of HA crystals in the direction of c-axis [3] .

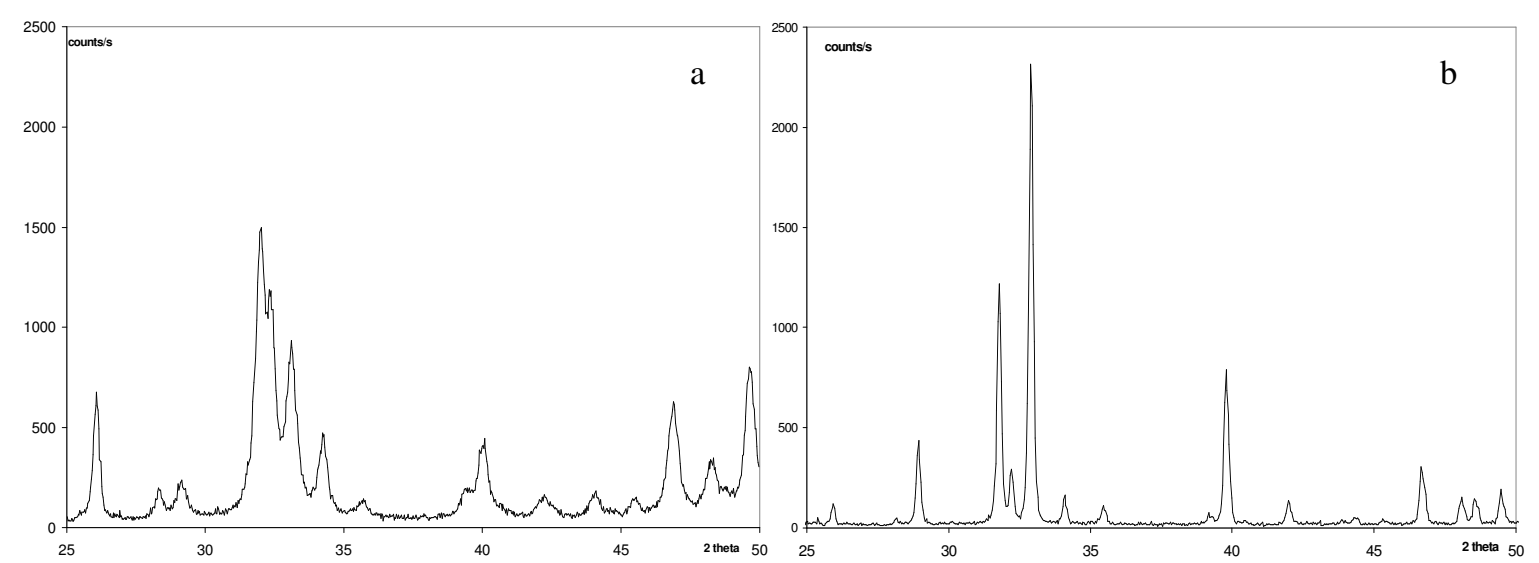

Figure 1: a) XRD pattern of commercial HA powder, b) XRD pattern of the HA whiskers prepared by molten salt synthesis.

The $\%$ relative density variation of the pure and composite HA samples are shown in Figure 2 as function of sintering temperature. The density varied almost linearly between 800 and $1150{ }^{\circ} \mathrm{C}$ and is almost completed (99\%) at $1150{ }^{\circ} \mathrm{C}$ for HA. The densification of the composite; however, started at temperatures above $1150{ }^{\circ} \mathrm{C}$. Relatively low density and high densification temperatures of the composites shown in Figure 2 are likely due to the high crystallinity and thermal stability of the whiskers, which resist to the atomic diffusion phenomenon. The hardness values of the pure and HA composites (Fig. 3) show well agreement with the density values given in Figure 2 within 800-1150 ${ }^{\circ} \mathrm{C}$. In this temperature range, the hardness of the pure HA increases with increasing sintering temperature, while the harness of the HA composite remains nearly constant. Sintering above $1250{ }^{\circ} \mathrm{C}$ however resulted in reductions in the hardness of the pure HA. This is likely due to the excessive grain-growth. Similar to the relative density, the hardness of the HA composite increases with increasing temperature above the sintering temperature of $1150{ }^{\circ} \mathrm{C}$. The maximum hardness value of the composite is $600 \mathrm{HV}$ and was determined $91 \%$ dense, $10 \%$ whisker reinforced composite. This maximum hardness value was higher than the hardness of the almost $100 \%$ dense pure HA, which clearly shows the potential of the whiskers for tailoring the strength of the HA. The hardness test could not be performed accurately since the $30 \%$ whisker reinforced composite contained relatively large pore sizes and significant amount of porosity.

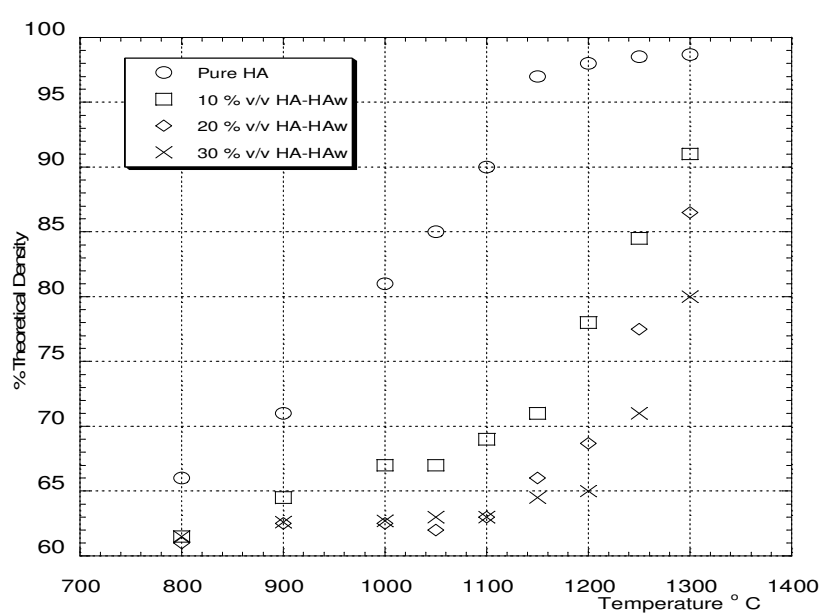

Figure 2. Sintering behavior of the pure and HA composite samples. 


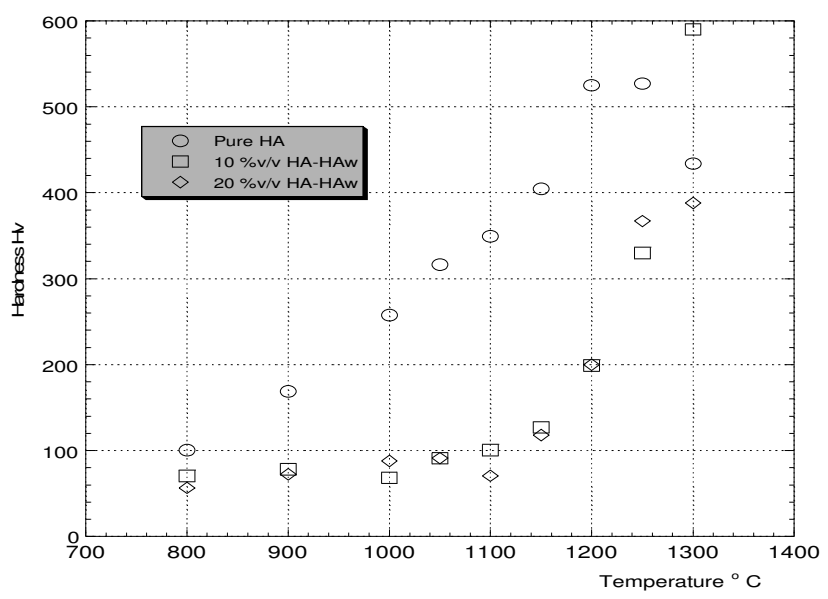

Figure 3. The variation of the hardness of the pure and composite HA with the sintering temperature.

The compression stress-strain curves of the pure and HA composites sintered at $1300{ }^{\circ} \mathrm{C}$ are shown in Figure 4. The effect of whisker-addition, as shown in this figure, is to increase compressive strength and fracture strain and therefore the toughness of the HA. It is also noted increasing whisker content from 10 to $30 \%$ has no significant effect of on the compressive strength. This is probably a branching effect of the reduced relative density at increasing whisker volume fractions. For comparison purposes, the compressive strength and modulus of the bone, pure HA and whisker reinforced composite HA are tabulated in Table 1. The modulus of the composite samples is very similar to those of bone and pure HA, but the compressive strength is nearly twice that of the bone and pure HA. Finally, the lower value of the modulus of the $30 \%$ whisker reinforced composite as compared with that of the $20 \%$ whisker composite (Table 1 ) is because of the lower relative density of the former.

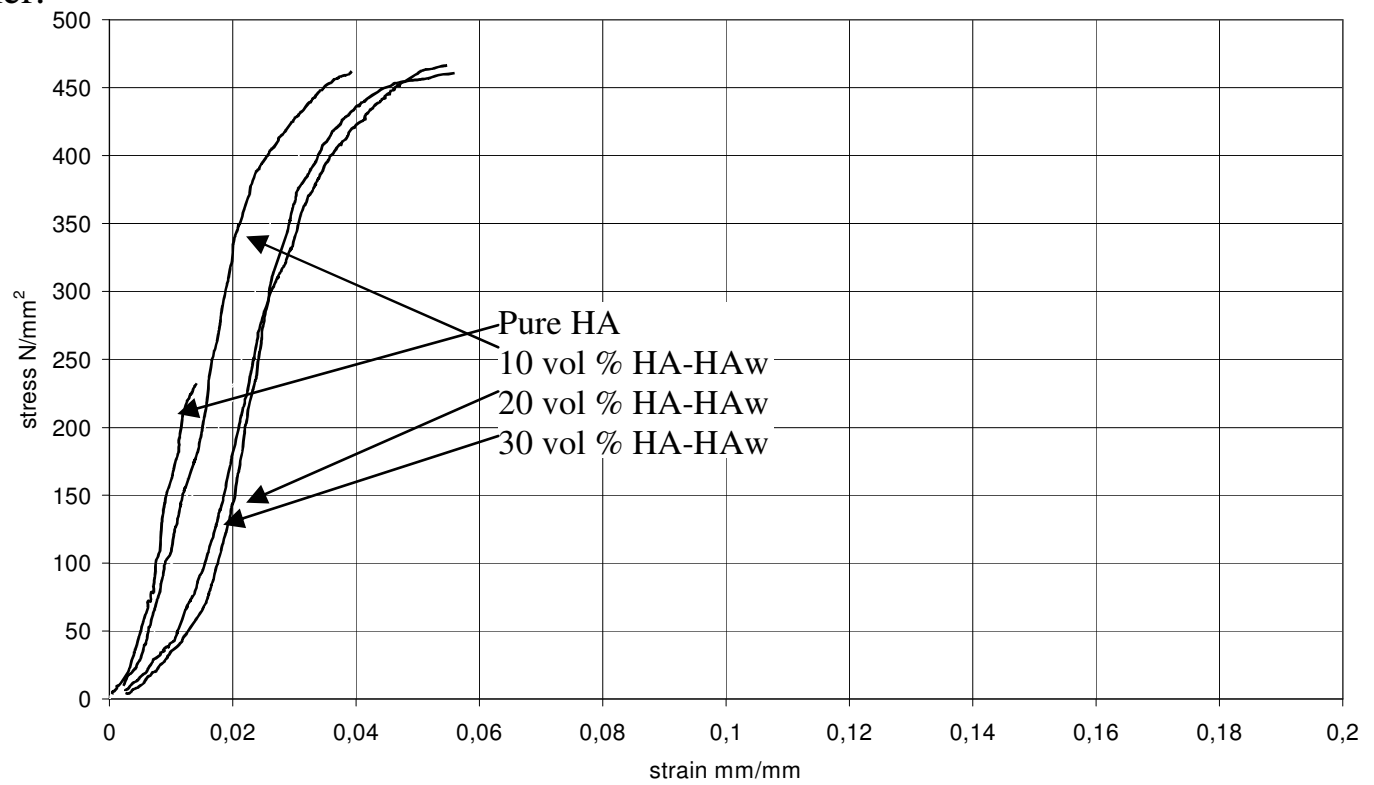

Figure 4. Stress-strain behavior of pure HA and HA-HA ${ }_{w}$ composites under compressive loading. 
Table 1: Comparison of the compressive strength and elastic modulus of the bone pure and HA composites.

\begin{tabular}{|c|c|c|}
\hline Material & $\begin{array}{c}\text { Compressive strength } \\
\text { (MPa) }\end{array}$ & $\begin{array}{c}\text { Elastic modulus } \\
\text { (GPa) }\end{array}$ \\
\hline Bone $[2,4]$ & $170-193$ & $14-18$ \\
\hline Pure HA & 240 & 12 \\
\hline Whisker composite & $460-470$ & $\begin{array}{ll}14 & (10 \%) \\
17 & (20 \%) \\
11.8 & (30 \%)\end{array}$ \\
\hline
\end{tabular}

The fracture surface of the pure HA shows a typical cleavage / brittle type of fracture (Fig. 4(a)). The features on the fracture surface of the composite: change of the crack path (Fig. 4(b)) and whisker-pullout (Figure 4(c)), also tend to conform the higher toughness of the composite as compared with pure HA.

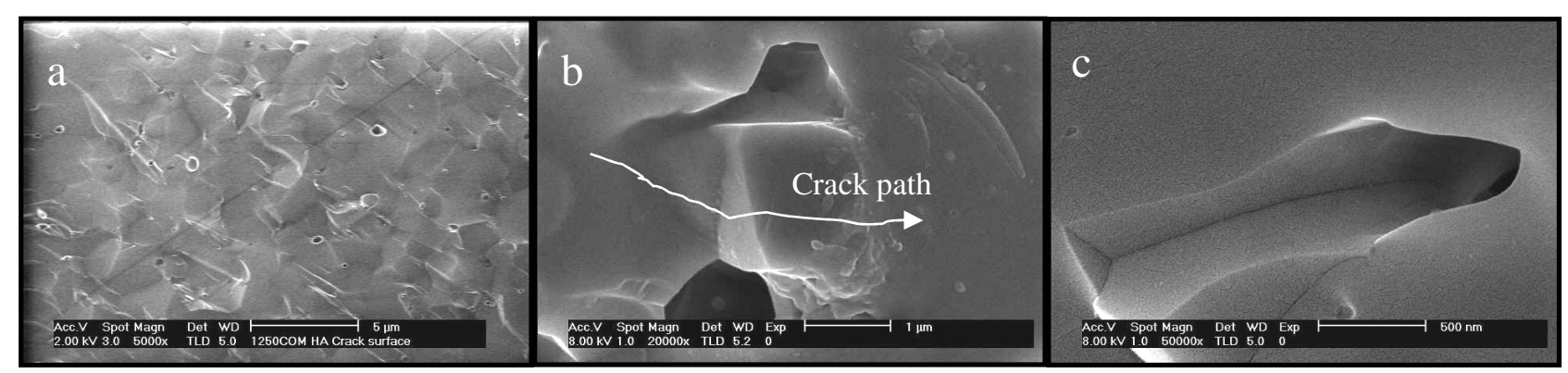

Figure 4. SEM micrographs of fracture surfaces a) pure HA sintered at $1200{ }^{\circ} \mathrm{C}$ and b, c ) $10 \%$ whisker reinforced composite sintered at $1300{ }^{\circ} \mathrm{C}$.

\section{Summary}

The sintering and mechanical behaviour of the pure HA and molten salt synthesis HA whisker reinforced composites were investigated. Sintering behaviour of the composites was determined to be quite different from that of the pure HA within the studied sintering range. In composites, significant densification started only after $1150{ }^{\circ} \mathrm{C}$, while pure $\mathrm{HA}$ reached to a $99 \%$ relative density at the same temperature. Compressive strength and fracture strain and hence fracture toughness increased with the addition of the HA whiskers. The microstructural investigation of the fracture surfaces of the composite indicated that crack path change and whisker pullout were the two main mechanisms of increased toughness in the composites.

\section{References}

[1] Suchanek W. Yashima M. Kakihana M. Yoshimura M., J.Am.Cer.Soc. 80(11), pp:2805-2813, (1997).

[2] SuchanekW. and Yoshimura M., J.Mater. Res.,13(1) pp:94-116, (1998).

[3] Taş C.A., J. Am. Ceram. Soc. 84 (2), pp:295-300, (2001).

[4] Hench L.L., MRS Bulletin, pp:13-19, May (1999). 


\section{Euro Ceramics VIII}

10.4028/www.scientific.net/KEM.264-268

Mechanical Properties of Hydroxyapatite Composites Reinforced with Hydroxyapatite Whiskers 10.4028/www.scientific.net/KEM.264-268.1985

\section{DOI References}

[1] Suchanek W. Yashima M. Kakihana M. Yoshimura M., J.Am.Cer.Soc. 80(11), pp:2805-2813, 1997). 10.1111/j.1151-2916.1997.tb03197.x 